\title{
Interfacing of Java 3D objects for Virtual Physics Lab (VPLab) Setup for encouraging Physics Studies
}

\author{
Prakash A. Bhadane ${ }^{1}$, S.M.Sabnis ${ }^{2}$, P. G. Kulkarni ${ }^{3}$ \\ 1(Department: Physics, Saraswati college of Engineering, plot no-46, sector-05, behind MSEB sub station, \\ Kharghar, Navi Mumbai-410210, Maharashtra, India,/University Name:-Author 1 is Ph.D. Scholar of Shri JJT \\ University, Vidyanagari, Churu Jhunjhunu Road, Chudela, District-Jhunjhunu, Rajasthan-333001.
}

\begin{abstract}
During this paper we target the combination of web accessible physics experiments (VPLabs) combined with the Sun's toolkit for making cooperative $3 D$ virtual worlds. Among such a cooperative setting these tools give the chance for academics and students to figure along as avatars as they control actual instrumentation, visualize natural phenomenon generated by the experiment, and discuss the results. Especially we'll define the steps of integration, future goals, yet because the price of a collaboration area in Wonderland's virtual world.
\end{abstract}

\section{INTRODUCTION}

The use of 3D virtual learning environments in education has big significantly over the past years and has captured the eye and interest of educators worldwide. This interest originally arose from educators' demands for a lot of versatile and communicative technologies to support the students' learning experiences. Despite fast development, several instructional application domains still exhibit a good want for improvement. If we concentrate on physics education from middle school through university, the most pedagogic drawback remains that students have nice issue in relating physics theory and equations to the phenomena they observe within the workplace or, indeed, within the everyday world. Maybe the foremost promising tools for raising students' ability to relate physics theory to discernible phenomena is that the use of $3 \mathrm{D}$ visual image.

\section{A. Java 3D Virtual Environments}

Virtual environments consist entirely of 3D elements, with that the user is ready to act inside the atmosphere. As an example, the user will enter and exit rooms, walk around buildings, and open drawers to visualize what's within. Will increase in desktop 3D special effects and network infrastructure were the 2 main technological advances that have enabled the event of 3D VEs and have usually augmented the potential of the planet Wide internet. Such environments offer the illusion of being immersed inside a 3D house, and change the user to perform actions and behaviors that are analogous to those she will initiate within the globe [16]. The very fact that users will gain expertise within the same method that they will within the globe opens new and fascinating opportunities for physics education. By increasing reality on the far side the bounds of the schoolroom in each time and area, the educator will enrich the student's learning expertise to ease understanding of abstract physics ideas [8]. Instead of making an attempt to recreate common face-to-face schoolroom settings, the aim of those environments is to enhance and expand schoolroom activities. Additionally to providing additional versatile learning resources than those out there in typical e-learning environments, VLEs additionally try and use the dimension to boost cooperative learning and supply active learning experiences impractical within the schoolroom.

A 3D simulation generated from a proper mathematical model embodying the laws of physics becomes a governable computer-generated analog of real-world objects or processes, that the scholar will use to grasp the behavior of advanced systems [2][4]. Together with visualizing physics phenomena and simulating their actions/ behavior, a 3D VLE additionally provides customary mechanisms for the user to move with the underlying physical/mathematical models. PC generated simulations will alter the scholars to match theoretical with experimental results, since they embody learning by doing [6].

\section{B. Collaborative Learning Environments}

While many VLE's for virtual physics experiments are designed as single-user environments, whereby learners accomplish experiments by themselves, recent approaches demonstrate that sharing experiences in an exceedingly cluster, discussing results along, and interacting with alternative participants will bring learners to the next accomplishment level [14]. Multi-user VLEs, conjointly referred to as cooperative VLEs (CVLE) [8] or virtual worlds [1], modify interactions not solely with objects however conjointly with alternative users. Multiple learners will explore an equivalent 3D cooperative atmosphere, will communicate with alternative learners, and might move their avatars through the house as if they were physically walking [5]. CLVEs have 
achieved the goal of virtual collaboration, thus they need the potential to supply vital influences on the 3D immersive virtual world approach [3].

The goal of our project is to supply a 3D CVLE for physics education within which students and educators are ready to work along in an exceedingly cooperative approach. Through the mixing of specific technologies and comes, the users of this immersive atmosphere use a 3D simulation to examine the important behavior of an actual remote physics experiment. The subsequent section of this paper can introduce the software package and technologies that are accustomed understand this cooperative learning house. We'll illustrate each this implementation in addition as our planned future work.

\section{BACKGROUND TECHNOLOGIES}

Our study is based on manifold pre-existing projects which personify virtual technologies. They each have their individualadvantages, and our objective has been to blend them into aninnovativemutualeruditionatmosphere.

\section{A. Project Wonderland}

Project Wonderland could be a 100 pe Java open supply toolkit for making cooperative 3D virtual worlds, developed by Sun Microsystems inc [12]. This multi-user immersive atmosphere incorporates 3D and PC graphics technology with digital media, to immerse and have interaction users within the same means 3D games grab and keep the eye of players. Wonderland offers students a virtual world among that to figure and learn along without the necessity for a separate collaboration tool [12].

As a result of the wonderland system is open-source; it is often extended by making new tools. Client/server architectures are similar temperament for game systems since the server will coordinate the state of all consumer instances. Besides the Dark-star server, Project Wonderland depends on any open-source comes developed by Sun Microsystems, together with Java3D [10], Project glass [4] and j-VoiceBridge [12].

\section{B. The VPLab Project:-}

The VPLab Project [7] built up at MIT endows an infrastructure for the drawing and allocation of Internet accessible lab experiments. It lets students and educators distantadmission to definiteapparatus in actual laboratories. Unlike conformistexperimentation facilities, the apparatuscan be shared and used anytime, anywere.

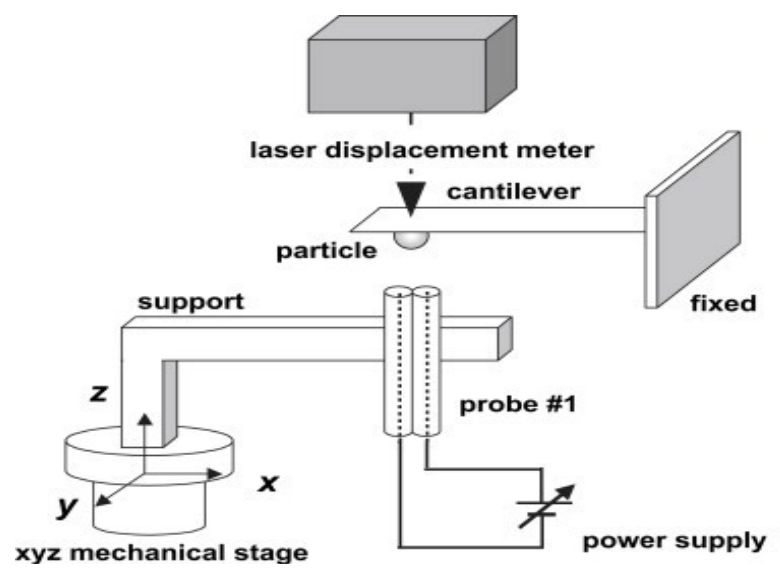

(Figure 1. Schematic representation of Force on a Dipole experiment)

Online laboratories impart the likelihood to increase the variety of experimentations that students employ in the course of their education.

\section{The Sun's Simulation System (Java 3D):-}

Sun's Simulationtoolkit [16] is an open source Java3D based mostly simulation toolkit, developed at the Massachusetts Institute of Technology (MIT) as a element of the 'Studio Physics' Technology Enabled Active Learning Project (TEAL) [16]. TEAL's major goal is to assist students create mentally phenomena and processes particularly in electromagnetism [7]. The approach of this project is to produce a mixture of lectures, science lab experiments and a spread of simulations in a very specially designed schoolroom to facilitate cluster interaction. Students usually work in teams of 3.

They're accustomed illustrate abstract physics ideas unreal by means that of a 3D model. To administer one example, magnetism fields and phenomena are usually not visible in real settings, however using such a simulation engine, students will read a synchronized visual image that renders a correct illustration of the invisible field lines. Student laboratory teams have the power to move with the model using their desktop input 
devices, management input values, so see however the simulation is established by their changes. Additionally, 3D simulations are often dole out as often as required to administer the scholars an improved understanding for physics ideas.

\section{Experimental Comparison}

The "Force on a Dipole" experiment developed at MIT combines associate VPLab interactive work and a SIM Line image. The experiment itself consists of a tiny low magnet suspended vertically by a spring within the center of 2 horizontally mounted coils. An electrical current could also be directed through the highest or, through each coil. A video camera is stationed to look at the position of the magnet (Fig.1).

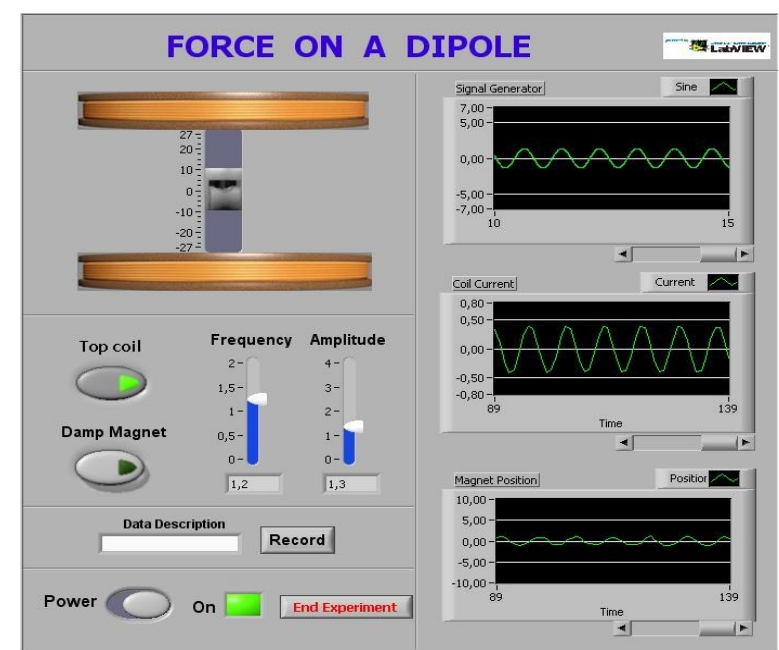

Figure 2. Lab VIEW application (Source: LabVIEW online)

This experiment is controlled by a LabVIEW [13] application ( Fig. 2 ), that permits users to tack the present amplitude and frequency of the present further on specify to that coils it's directed. The applying displays the input current, the present within the high coil and therefore the position of the magnet. Additionally, users could request that information be collected, and an overseas method could request that the experiment information be streamed to that using TCP/IP.

Using the SIM Line Toolkit, a specialized application [9] was designed to buy the experiment information stream and to use the info to drive a period of time image of the experiment on the student's laptop. Thisview suggests that the Lab VIEW interface allows remote access to regulate the experiment whereas the SIM Line package creates a synchronized 3D simulation of the flux lines as an instance the interaction of the stationary current carrying coil with the suspended static magnet ( Fig. 3 ).

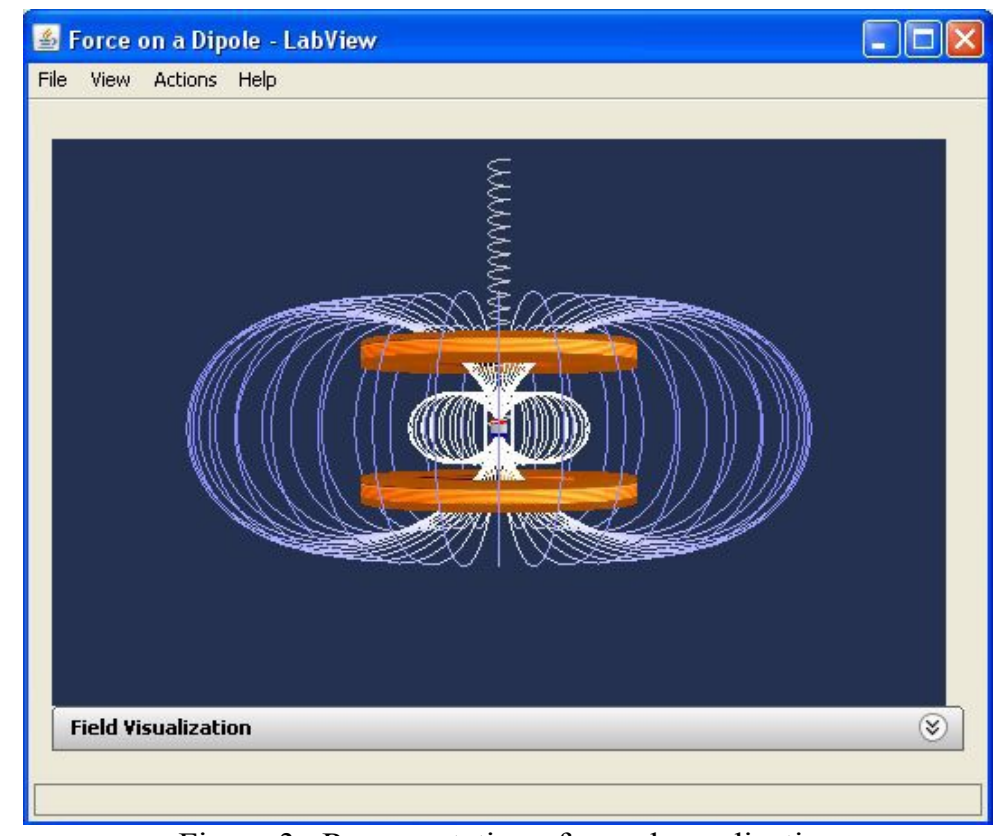

Figure 3. Representation of sample application 
The combination of those comes provides a robust tool to boost understanding of abstract physics ideas using active and passive visualizations, particularly in areas wherever we will create the "unseen seen", as in electromagnetism. These instructional advantages are currently accessible to students and educators round the world. In making an attempt to increase the established advantages of the current "Force on a Dipole" project, the present team formed the concept of cooperative immersive surroundings for physics education. The TEAL Studio Project already reflects this approach by providing physical cooperative learning surroundings. However it assumes that students are physically collocated and might physically act. The present project aims to realize identical goal once that's insufferable. Within the next section, we describe the conclusion of these cooperative surroundings using Project Wonderland tools and also the integration of the "Force on a Dipole" project.

\section{VIRTUAL LAB ENVIRONMENT}

We will 1st make a case for the fundamentals of Wonderland's infrastructure so as to offer a far better understanding of however image and interaction add Wonderland. At the time we started with our analysis work, Project Wonderland's unharness 0.4 was the stable version to form a 3D world area. This can be the version on that this project is predicated though we expect to migrate it to future releases of the Wonderland atmosphere.

\section{A. Wonderland's Cell Architecture:-}

Wonderland organizes the 3D virtual world in cells. A cell represents a volume of house within the world, like a part of a scene or an object. Avatars are pictured through cells. The cell consists of each a consumer and a server object. The server part, instantiated by the sport server Project Dark star, consists of one category that maintains the shared state of the cell across all consumer participants. The client-side half is accountable for rendering the cell in-world either by loading an art resource or by drawing objects directly using the Java3D apis. Cells divide the world's Java3D scene graph into network edoptimized chunks. as an example, the World Root Cell, that represents the complete world, might encompass many space Cells, and every of those space Cells might encompass an Application Cell, Avatar Cell, etc.

Communication between consumer and server is complete through client-server messages. If a consumer action causes a client-cell method natural action, messages are going to be sent from consumer to server to update the server-side instance of this cell. The absolute 3D-coordinates of a cell's origin at intervals the globe additionally because the name of the category to that the cell object belongs is provided in an XMLformatted cell descriptor file at intervals the Wonderland-File-System (WFS). The WFS records instances of cells that are supplementary to a world. When the Project Wonderland-Server is started or reloaded, the WFS scan and an instance of a cell is formed on the server for every XML cell descriptor within the WFS. When a consumer connects to the server the client-side cells are created for every cell presently within the server.

\section{B. ForceonaDipole Cell}

In our project, integration the TEAL simulation of the Force on a Dipole experiment needed the creation of a brand new cell kind with server-side and client-side categories. The server-side cell SIM Line needs to communicate with the client-side SIM Line when the client starts so as to setup the cell's properties and to update them throughout the applying runtime. The foremost tasks of the clientside SIM Line, besides providing access to the cell's scene graph, is to inform the server of the user interactions and to method the setup and update messages from the server-side cell.

\section{Integration of the experimental Model}

SIM Line simulations are Java categories that have a well outlined interface (TSimulation) and contain 3 major elements, the simulation engine, the rendering engine, and also the interface. When running a simulation on the desktop or as and application, the simulation is run at intervals a TEAL framework that is to blame for loading the simulation and presenting the computer program. Simulations weren't designed to run outside of a framework, for this project the SIM Line is employed as the framework.

The SIM Line render engine is enforced as a Java3D-based viewer that manages the simulation's scene graph. Thejava interfacing reality created it attainable to integrate the both technologies with a number of changes to the SIM Line design.

When the Wonderland consumer is started, the SIM Line- Cell creates an instance of the Force onADipole simulation. The scene graph out of it should be integrated into the complete scene graph of Wonderland by attaching it to the cell's scene graph node. Since the SIM Line scene graph was hooked up to the SIM Line viewer, it's to be 'detached' before this will be done. Finally this complete subgraph is then said to be board Wonderland's world.

The simulation engine maintains a model of this state of all simulated objects and perpetually integrates the interaction of all objects within the simulation. For performance reasons the simulation engine runs at 
intervals its own thread. to regulate the looks of the 3D simulation within Wonderland, the particular experiment should be started before the simulation will hook up with the information stream.

\section{Connection to the actual Experiment}

The VPLabs framework provides access to the remote VPLabLab Server that launches the interactive Lab VIEW application. For this purpose, Wonderland supports a graphical desktop sharing system, known as Virtual Network Computing (VNC), to manage another pc remotely. A VNC viewer, integrated as a cell in Wonderland, will connect with a VNC server on identical or another pc. The VNC server, in our context, is found on identical server wherever the Lab VIEW software system is running. Within the world a VNC viewer is employed to show the graphical user interface front panel of the Lab VIEW application. User interaction is provided through keyboard or mouse events, that are transmitted from the pc on that the Wonderland consumer is running to the pc wherever the LabVIEW application is hosted. Multiple clients could connect with a VNC server at identical time. Therefore, avatars have the flexibility to launch and move with the important experiment via the VNC viewer even as students within the room version of TEAL will management the frequency and amplitude of the present.

\section{E. Starting the simulation in world}

After the important experiment is started, avatars will currently begin the virtual illustration of the experiment in world. Within the TEAL framework, this action is triggered by choosing a menu item, however inside Wonderland zero. 4 the simulation's program parts don't seem to be out there. The training area should offer a button, enforced as an Inter-Cell communication cell. Once the button is ironed, a message is shipped to the button's server-side cell that then sends a message to the server-side SIMLine-CellGLO. A message is shipped from the server-side cell to every of the consumer cells requesting that the client's simulation model connect with the LabVIEW Application, every simulation opens an information socket to the LabVIEW application.

To replicate the result of the user's input on the simulation in Wonderland, the world's scene graph should be updated. When beginning the socket, we tend to connect once more to the particular branch cluster of the rendering engine and append it to the world's scene graph. So as to update the consumer itself and every one alternative to replicate the user input within the simulation, the SIMLineCell sends a message to the SIMLineCellGLO. This server aspect implementation has the vital task of relaying the message to all or any client.

When the client-side illustration of the beginning button is at the start pushed, the server cell should begin the affiliation on all alternative client cells. Conversely, if the button is ironed once more, clients have to be compelled to learn to prevent the socket so as to finish the simulation's affiliation. To represent application state, the button can address red once it's pushed to begin the simulation, and to inexperienced when pushed to prevent the simulation.

\section{F. Real-time experimentation}

For the Force onADipole application there's additionally a digital camera provided, that isn't a part of the appliance however access to the digital camera is expedited by the VPLab Service-Broker. Wonderland additionally permits students to stream the actions of the important hardware, completed in an exceedingly separate cell.

This cell facilitates a video screen in world wherever avatars are able to see the operating experiment via the network camera. The desired camera within the 'video-cam-wlc. xml' is that the camera, however it's doable to feature support for alternative Axis network cameras, just like the Axis 211 we tend to are using. The video is streamed to all or any purchasers and compete at the same time within the browser of all avatars within the same world, as long as the actual experiment continues to be connected. Control buttons enable the avatars to terminate and management the video.

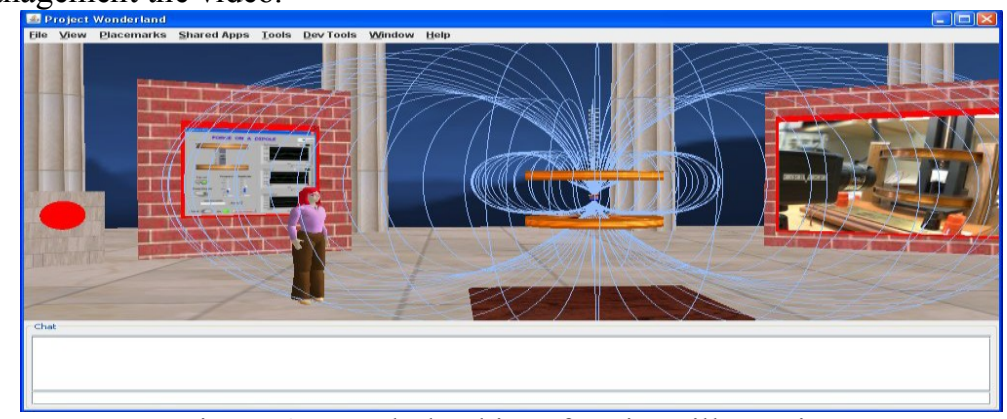

Figure 4. Wonderland interface java illustration 
The present state of our work could be an operating cooperative virtual setting in Wonderland version 0.4 that presently provides nearly the total practicality of the force on a dipole experiment.

For the longer term, the set up for this scientific research is to port from 0.4 to 0.5 to realize a richer 3D expertise among the planet. Wonderland is moving from a Java3D rendering engine to JMonkeyEngine (JME) [11] that ought to cure the restrictions obligatory on Wonderland by the visual and style constraints of Java3D. JME could be a full featured 3D game engine that is already in use by skilled game firms. JME ought to give higher performance additionally as higher graphics capabilities. This can need replacement the Java3D rendering engine employed by SIMLine and a design of the SIMLine 3D viewer. An extra vital modification in Wonderland 0.5 can replace the prevailing inflexible avatar system with a contemporary progressive avatar system together with new bone models and advanced skinning options, which can bring the looks of an avatar to the next level.

Finally, version 0.5 can give embedded swing practicality for general program construction. With this feature we get the power to make our own Swing-based program to act with the experiment rather than employing a VNC-viewer to indicate the LabVIEW application.

Our goal is to support the TEAL simulation's program elements with this Swing feature. Once porting to 0.5, successive step within the future are extending the educational area by populating the virtual learning setting with additional simulations.

\section{CONCLUSION}

In this paper, we've started our analysis work and vision for cooperative, simulation-based learning in desktop 3D virtual environments. The arrival of the desktop variant of 3D virtual environments has created the technology wide accessible and has dramatically reduced the prices of the mandatory hardware.

The obvious advantage of virtual laboratories lies in reducing the quantity of pricey physical laboratories that has to be maintained and in permitting students remote access at any time. Support for teaching laboratories is important, and then there's a true potential for permitting widespread use of virtual laboratories. The utilization of such laboratories during a cooperative virtual learning setting improves the potential impact of virtual laboratories by permitting collaboration between students and academics and between students themselves. It conjointly permits international collaboration between students by forming learning groups across continents so as to unravel issues.

Besides providing a technical answer to understand a cooperative learning setting, we believe that it's crucial to determine a brand new learning culture with horizontal communication structures. Active participation and meaty discussion are the cooperative values we wish to support in our virtual setting.

\section{REFERENCES}

[1] R.A. Bartle, "Designing Virtual Worlds", New Riders Publishing, Indianapolis, 2004, Chapter 1, pp. 2.

[2] Bouras, C., \&Tsiatsos, T. (2006). Educational virtual environments: design rationale and architecture. Multimedia Tools and Applications, 29, 2, 153-173. (doi:10.1007/s11042-006-0005-7)

[3] S. Bronack, R. Sanders, A. Cheney, R. Riedl, J. Tashner, \& N. Matzen, "Presence pedagogy: Teaching and learning in a 3D immersive world”. International Journal of Teaching and Learning in Higher Education, 2008, 20(1), pp. 59-69.

[4] Y.S Chee, C.M. Hooi, "C-Visions: Socialized Learning through Collaborative, Virtual, Interactive Simulations". Published in Proceedings of CSCL 2002: Conference on Computer Support for Collaborative Learning, Boulder, CO, USA, pp. 687-696.,

[5] Cherney et al., "Teaching and Learning in a 3D Immersive World: The AETZone Model", 2007.

[6] B. Dalgarno, "The Potential of 3D Virtual Learning Environments: A Constructivist Analysis", Electronic Journal of Instructional Science and Technology, 5(2), pp. 3-6, 2002.

[7] Y.D. Dori, J. Belcher, "How does technology-enabled active learning affect undergraduate students' understanding of electromagnetism concepts?" The Journal of the Learning Sciences, 14(2), pp. 243-279., 2005.

[8] D. Economou, W. L. Mitchell, T. Boyle, "Requirements elicitation for virtual actors in collaborative learning environments", Computers \& Education 34, pp. 225-239, 2000. (doi:10.1016/S0360-1315(99)00047-0)

[9] Force On Dipole, Retrieved May 20th, 2009

[10] Java3D, Retrieved May 20th, 2009 from http://java.sun.com/javase/technologies/desktop/java3d/

[11] jMonkeyEngine, Retrieved May 20th, 2009 from http://www.jmonkeyengine.com

[12] jVoiceBridge, Retrieved May 20th, 2009 from https://jvoicebridge.dev.java.net/

[13] LabVIEW, Retrieved May 20th, 2009 from http://discoverlab.com/References/WP2238.pdf

[14] Moriarty P J, Gallagher B L, Mellor C J and Baines R R 2003 Am. J. Phys. 71 1062-74

[15] Doyle F J III, Gatzke E P and Parkers R S 1998 Comput. Appl. Eng. Educ. 6 181-91

[16] Foley E L, Candela D, Martini K M and Tuominen M T 1999 Am. J. Phys. 67 389-93 